OPEN ACCESS

Edited by: François Billaut, Laval University, Canada

Reviewed by:

Zhaowei Kong,

University of Macau, Macau

Dale R. Wagner,

Utah State University, United States

*Correspondence:

Morten Kristoffersen morten.kristoffersen@hvl.no

Mkr@hvl.no

Specialty section:

This article was submitted to

Exercise Physiology,

a section of the journal

Frontiers in Physiology

Received: 18 June 2019 Accepted: 16 August 2019

Published: 28 August 2019

Citation:

Kristoffersen M, Sandbakk Ø, Rønnestad BR and Gundersen H (2019) Comparison of Short-Sprint and Heavy Strength Training on Cycling Performance.

Front. Physiol. 10:1132. doi: 10.3389/fphys.2019.01132

\section{Comparison of Short-Sprint and Heavy Strength Training on Cycling Performance}

\author{
Morten Kristoffersen ${ }^{1,2 *}, \varnothing y v i n d$ Sandbakk ${ }^{2}$, Bent R. Rønnestad ${ }^{3}$ and Hilde Gundersen ${ }^{1}$ \\ ${ }^{1}$ Department of Sport, Food and Natural Sciences, Western Norway University of Applied Sciences, Bergen, Norway, \\ ${ }^{2}$ Centre for Elite Sports Research, Department of Neuroscience and Movement Science, Norwegian University of Science \\ and Technology, Trondheim, Norway, ${ }^{3}$ Section for Sport Science, Inland Norway University of Applied Sciences, \\ Lillehammer, Norway
}

Purpose: To compare the effects of short-sprint training (SST) and heavy-strength training (HST) following a 4-week strength-training period on sprint and endurance capacities in well-trained cyclists.

Methods: Twenty-eight competitive cyclists (age $29 \pm 6$ years) with maximal oxygen uptake $\left(\dot{\mathrm{V}} \mathrm{O}_{2 \max }\right)$ of $61.1 \pm 5.9 \mathrm{~mL} \cdot \mathrm{min}^{-1} \cdot \mathrm{kg}^{-1}$ participated. After a 4-weeks preparation strength-training period, the participants were randomized to add either HST or SST to their usual endurance training for the subsequent 6 weeks. Body composition, $\dot{\mathrm{V}}_{2 \max }$ and power output at blood lactate concentration ([La $]$ ) of $4 \mathrm{mmol} \cdot \mathrm{L}^{-1}$, as well as a 100 min cycling test including 6 and 30 -s sprints, 60 min cycling at $\left[\mathrm{La}^{-}\right]$of $2 \mathrm{mmol} \cdot \mathrm{L}^{-1}$ and 5-min all-out cycling were performed before the 4-week preparation strengthtraining period, and before and after the 6-week intervention period. In addition, 1 repetition maximum (RM) in half-squat and 55-m maximal sprints on the cyclists' own bikes were measured before and after the 6-week intervention.

Results: SST was superior to HST in 6-s sprint performance, both in a fresh state $(4.7 \pm 2.6 \%$ vs. $1.1 \pm 3.5 \%)$ and after prolong cycling $(6.1 \pm 1.8 \%$ vs. $1.8 \pm 4.2 \%)$, in 30 -s sprint $(3.7 \pm 2.8 \%$ vs. $1.3 \pm 2.5 \%)$ and in 55 -m seated sprint on own bike $(4.3 \pm 2.1 \%$ vs. $0.2 \pm 1.8 \%$ ) (all $p<0.002)$. HST induced a larger 1RM improvement in the half-squat test than SST $(9.3 \pm 3.6 \%$ vs. $-3.9 \pm 3.8 \% ; p<0.001)$. No group differences were revealed in the 5 -min all-out test, $\dot{V}_{2}$ max , power output at $4 \mathrm{mmol} \cdot \mathrm{L}^{-1}$ $\left[\mathrm{La}^{-}\right]$, or in gross efficiency.

Conclusion: SST led to a greater increase in average and peak power output on all sprint tests compared to HST, whereas HST led to a greater increase in maximal strength. No group differences were found in relative changes in endurance capacities. Altogether, our results show a high degree of specificity in the adaptations of both SST and HST.

Keywords: road cycling, short-sprint training, strength training, concurrent training, power output, trained subjects 


\section{INTRODUCTION}

Road cycling is a demanding endurance sport, with performance mainly determined by maximal aerobic power, the ability to utilize a large fraction of maximal aerobic power over long-lasting competitions and cycling efficiency (Jeukendrup et al., 2000; Faria et al., 2005). In addition, the ability to perform breakaways, to close gaps, and to sprint fast in the finish-sprint are important to win races (Faria et al., 2005). Although endurance training is undoubtedly the most important component in cyclists' training regimen, training with the goal to increase the capacity to produce high-power output for a relatively short duration through heavy strength and sprint training could be beneficial. In fact, especially during the preseason, many competing cyclists include strength training in addition to the usual endurance training with the goal to increase the capacity to produce high-power output. A strengthtraining period usually starts with a preparation period focusing on building basic strength capacity through high volume and medium load, followed by a period with heavy strength training (HST) to increase maximum strength through lower volume and higher load. Finally, there is a sport specific high power strength period focusing on maximal power development (Kraemer and Ratamess, 2004; Fleck, 2011). It has been shown positive effects of HST on various factors related to cycling performance, without any negative interference effects on the endurance capacity (Koninckx et al., 2010; Ronnestad et al., 2010a,b, 2011, 2015a, 2016; Aagaard et al., 2011; Ronnestad and Mujika, 2014; Vikmoen et al., 2016). To the best of our knowledge, the effect on cycling performance of going directly from the basic strength-training phase to the sport specific maximal power development phase has not been compared with the traditional approach of moving from the basic strength phase to the maximum strength phase.

Although many road cyclists regularly carry out short-sprint training (SST) in addition to the usual endurance training during the pre-season, only a few studies have examined the effects of this training method. Specifically, two previous studies found that only 2 weeks of SST, involving 6-s sprint intervals, improved peak power output (PPO) and $10 \mathrm{~km}$ time trail cycling performance in triathletes (Jakeman et al., 2012) and in physically active men (Lloyd Jones et al., 2017). Another study conducted with trained students found that 7 weeks of SST, performed as 5-s sprint intervals, improved PPO and total work during 30-s allout cycling (Linossier et al., 1993). Consequently, both HST and SST are common methods in competitive cyclists. However, to date, no studies have compared the effects of HST and SST during the pre-season on sprint and endurance capacities. Therefore, the main aim of the present study was to compare the effects of 6-week HST or SST after a 4-week preparation strength period on sprint and endurance capacities in trained cyclists. In addition, we compared whether the possible effects from these types of training could be transferred to sprinting ability on the cyclists' own bike. We hypothesized that a larger improvement in sprint performance would occur after SST (as compared to HST), while we expected no differences between SST and HST in endurance capacities.

\section{MATERIALS AND METHODS}

\section{Participants}

Thirty-two participants (28 men and 4 women) were included in the study, but due to injury and illness, four participants withdrew. Thus, in total, 26 men and 2 women (age $29.6 \pm 0.6$ years; height $183 \pm 7 \mathrm{~cm}$, weight $79.3 \pm 9.0 \mathrm{~kg}$ ) fulfilling at least two of Jeukendrup's criteria for trained road cyclists completed the study (Jeukendrup et al., 2000).

The Regional Committee for Medical and Health Research Ethics in Western Norway evaluated the study to ensure that it did not include any medical or health-related ethical concerns. The study was then approved by The Norwegian Data Protection Authority. All participants gave their written, informed consent to participate in the study, which was completed according to the Declarations of Helsinki.

\section{Experimental Design}

A pre-post design was used in this study (Figure 1). Participants were tested before and after a 6-week training intervention. After a 4 weeks preparation strength period, participants were randomized either to implement SST $(n=16)$ or HST $(n=16)$ two times a week (in addition to their regular endurance training). All cyclists (except three from the SST group and one from the HST group) completed at least 10 of the 12 SST or HST sessions during the 6 -week training intervention, and had done at least 6 out of 8 HST sessions in the 4-week preparation strength-training period. Preliminary analysis showed no differences between groups for any of the variables before start of the 6-week intervention (Table 2; all $p>0.05$ ).

\section{Strength-Training Protocol}

A daily undulating periodization HST program (Rhea et al., 2002) based on previous studies reporting significant improvement in strength and cycling performance in welltrained cyclists was used (Ronnestad et al., 2010b, 2015a, 2016). The strength exercises included half-squat in a Smith machine, leg press with one foot at a time, one-legged hip flexion, and toe raise (Ronnestad et al., 2015b). The HST was conducted with the intention of maximally accelerating the load during the concentric phase, while the eccentric phase was performed more slowly.

In the 4-week preparation strength training period, before the intervention, all participants performed 3 sets of 10RM in the first weekly session and 3 sets of 6RM in the second weekly session during the three first weeks, and 3 sets of $8 \mathrm{RM}$ and 3 sets of 5RM the last week. During the 6-week intervention, the participants in the HST group performed 3 sets of $8 \mathrm{RM}$ in the first weekly session and 3 sets of 5RM in the second weekly session for 2 weeks, and 3 sets of $6 \mathrm{RM}$ and 3 sets of $4 \mathrm{RM}$ during the four following weeks. Rest between sets and exercises was 2 min. To ensure proper technique and load, an investigator supervised all participants during the first week in the preparation period and all participants in the HST group at the first session in the 6-week intervention period. 


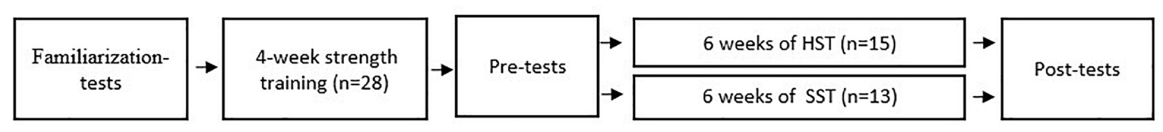

FIGURE 1 | An overview of the experimental design. HST, heavy strength training; SST, short-sprint training.

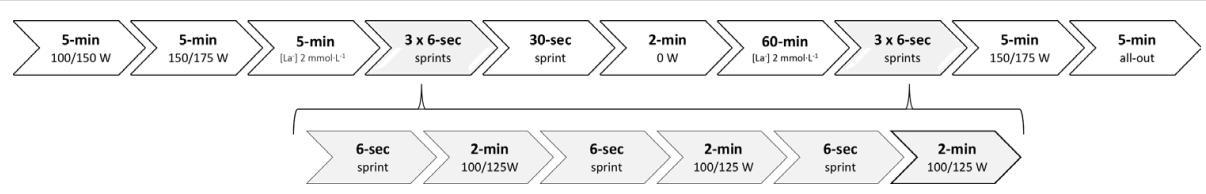

FIGURE 2 | An overview of time and load in the different phases of the 100 min cycling test. W, watt (female/male); [La ${ }^{-}$ 2 mmol. L $^{-1}$, load at blood lactate concentration of $2 \mathrm{mmol} \cdot \mathrm{L}^{-1}$ measured in blood lactate profile test performed before the first test period. 6-s sprints and 2 min rest between sprints performed in fresh state and after prolonged cycling are marked in gray.

The participants in the HST group were encouraged to continuously increase their RM loads throughout the intervention period and to record load $(\mathrm{kg})$, repetitions, and sets performed during each session into an electronic form after each session. They were also encouraged to complete the workouts with another participant from the HST group.

\section{Short-Sprint Training Protocol}

The SST program was based on a recent study conducted in our laboratory (Kristoffersen et al., 2018). All sprints in the SST sessions were conducted using an air-braked cycle ergometer (WattBike Ltd., Nottingham, United Kingdom) in a seated position, from a standstill start, with the preferred leg chosen by the participant. All the participants got the cycle ergometers individually adjusted before entering the study. Moreover, the pedaling resistance applied to the sprints was individually adjusted in the first SST session, where each participant performed at least three 6-s seated sprints at different resistance levels to ensure that the participant achieved the highest possible power output at a cadence of 110-120 rpm (Hopker et al., 2010; Herbert et al., 2015). The SST session consisted of 15 min warm-up, followed by three sets of four intervals with maximum effort (separated by 2 min of active recovery between intervals), and $5 \mathrm{~min}$ active recovery between sets of cycling at $70 \%$ maximal heart rate $\left(\mathrm{HR}_{\max }\right)$. To reflect practise, a daily undulating periodization SST program with progressive overload by increasing the duration of the sprint intervals every second week was used. During the first 2 weeks, the first and second weekly sessions consisted of 4- and 6-s sprints, respectively. The subsequent 2 weeks consisted of 7and 5-s sprints, and the final 2 weeks consisted of 8- and 6-s sprints. All sprint sessions were monitored and transferred to the Training Peaks diary through the participants' bike computers.

\section{Testing Procedures}

Each of the test periods, including the familiarization tests before the 4-week preparation strength training period, consisted of two separate test days: Day (1) Measurement of body composition, and a stepwise incremental cycle test to determine the power output at $\left[\mathrm{La}^{-}\right]$of $2 \mathrm{mmol} \cdot \mathrm{L}^{-1}$, followed by a $\dot{\mathrm{V}} \mathrm{O}_{2 \max }$ test.
Day (2) A 100-min cycling test, including 6- and 30-s maximal sprints, and a 5-min all-out test (Figure 2). In addition, a 1RM in half-squat and 55-m maximal sprint on the cyclists' own bikes were included on a third test day before and after the 6-week intervention.

All participants were instructed to abstain from strenuous exercise for $48 \mathrm{~h}$ before the tests, and to consume the same type of meal before each test day. There was a minimum of $48 \mathrm{~h}$ between test days, and all tests were conducted at the same time of day to avoid any possible effects of the circadian rhythm. All tests in the laboratory (day 1 and 2) were performed under uniform environmental conditions (17$19^{\circ} \mathrm{C}$ ) on the same electromagnetic bike (Lode Excalibur Sport, Groningen, Netherlands), which was adjusted according to each cyclist's preferences. On day 3, the 55-m sprint tests were performed under uniform environmental conditions $\left(11^{\circ} \mathrm{C}\right)$ in an indoor garage.

Test procedures for the body composition (bioelectrical impedance) analysis, the incremental lactate profile test and the $\dot{\mathrm{V}} \mathrm{O}_{2 \text { max }}$ test conducted in the present study are described in detail in a previous study (Kristoffersen et al., 2018). The remaining tests are described in detail below.

\section{0-min Test}

The 100-min cycling test protocol was completed using the Lode Ergometry Manager (LEM 10 Software, Germany), where power output was continuously registered throughout the entire test (Figure 2).

Each participant performed the familiarization-, pre- and post-tests with the same power output in each submaximal exercise phase. 6- and 30-s sprints were performed in a Wingate mode. The load was calculated from the participant's total body mass at baseline, and set to 0.8 (30-s) and 1.0 (6s) $\mathrm{Nm} \cdot \mathrm{kg}^{-1}$ body mass in men and to $0.8(6-\mathrm{s})$ and 0.77 (30-s) $\mathrm{Nm} \cdot \mathrm{kg}^{-1}$ body mass in women. The power output at $\left[\mathrm{La}^{-}\right]$of $2 \mathrm{mmol} \cdot \mathrm{L}^{-1}$ was determined with linear regression between the nearest data points below and above $2 \mathrm{mmol} \cdot \mathrm{L}^{-1}$ during the first lactate profile test at familiarization and this absolute value was used as the $2 \mathrm{mmol} \cdot \mathrm{L}^{-1}$ workload during all subsequent tests. 
The 5 -min and $60-\mathrm{min}$ phases at $\left[\mathrm{La}^{-}\right]$of $2 \mathrm{mmol} \cdot \mathrm{L}^{-1}$ were performed with the ergometer in a cadence-independent mode. All sprints were completed seated, starting with a cadence of 50-60 rpm. The 6-s sprints were separated by 2 min of active recovery (125 $\mathrm{W}$ in men and $100 \mathrm{~W}$ in women), with a 2 -min recovery without load after the 30 -s sprint. The best 6 -s sprint before and after prolonged cycling was used in the statistical analyses. The 5-min all-out cycling was performed with the ergometer in cadence-dependent mode (linear mode), where the power output increases with increasing cadence according to the following formula: $\mathrm{W}=L \times(\mathrm{rpm})^{2}$, where $\mathrm{W}$ is the power output, rpm is the cadence, and the constant $(L)$ determines the electronic gearing of the system. Here, it was set to 0.042 in men and 0.035 in women.

To calculate gross efficiency (GE) in fresh state (i.e., after 5 min cycling at $125 \mathrm{~W}$ in men and $100 \mathrm{~W}$ in women, followed by $5 \mathrm{~min}$ at $175 \mathrm{~W}$ and $150 \mathrm{~W}$, respectively) and after prolonged cycling, $\mathrm{VO}_{2}$ and respiratory exchange ratio (RER) were measured between the 2 nd and 4 th minutes in the phase before the 6-s sprint in fresh state, and between the 50th and 53rd minutes in the 60-min phase (Figure 2). Expired gases were sampled continuously, and average values for every $30 \mathrm{sec}$ were used in the calculations. $\mathrm{VO}_{2 \text { max }}$ and RER were measured using a computerized metabolic system with a mixing chamber (Oxycon Pro, Erich Jaeger, Hoechberg). The work rate was then divided by the metabolic rate to calculate GE (Skovereng et al., 2018). The participants were allowed to consume sports drinks in order to maintain fluid balance and to mimic race conditions. Volume and time of consumption were noted during the first test, and the same procedure was repeated in the following tests.

\section{RM in Half Squat}

$1 \mathrm{RM}$ in half squat was measured in a Smith machine (TKO, Houston, United States). The test was preceded by a 15-min warm-up on a cycle ergometer (WattBike Ltd., Nottingham, United Kingdom). Following the warm-up, the participants performed a standardized protocol, consisting of three sets with gradually increasing load and decreasing number of repetitions. The depth of the squat was set to approximately $90^{\circ}$. The squat depth and the feet placement were monitored and marked to ensure similar positioning at each test (Ronnestad, 2009). The first $1 \mathrm{RM}$ attempt was performed with a load approximately $5 \mathrm{~kg}$ below the predicted 1RM. After each successful attempt, the load was increased by $2.5-5 \mathrm{~kg}$.

\section{5-m Sprint}

Ten participants from the HST group and eight participants from the SST group performed the 55-m sprint test. Testing was performed on the participants' own bikes, and the participants were instructed to use the same bike with the same setting in each test. Before testing, the participants performed a 15min warm-up on an ergometer with the same load as the first $15 \mathrm{~min}$ in the 100 -min test. All participants performed 3 seated and 3 standing sprints, with 2 -min recoveries between each. Before the beginning of each attempt, the participant clipped her/his pedal, and an experimenter then maintained the participants starting position. The test was carried out with a static start, and the participants started at a verbal command. Times were measured using wireless timing gates (Muscle Lab, Ergotest Technology, Langesund, Norway). The best $55-\mathrm{m}$ times in seated and standing sprints were used in the subsequent analyses.

\section{Training Load and Intensity}

All training during the 4-week strength training period, and during the 6-week intervention period was monitored and transferred into each participants' training diary account (Training Peak, Peaksware LLC, Lafayette, CO, United States) and analyzed with the analyzing program WKO4 (Peaksware LLC, Lafayette, CO, United States). There were no difference between the SST and HST group in neither weekly logged training duration (4-week strength training period: $12.6 \pm 7.6 \mathrm{~h}$ vs. $11.8 \pm 5.8 \mathrm{~h}$, intervention period: $14.4 \pm 8.6 \mathrm{~h}$ vs. $14.1 \pm 8.4 \mathrm{~h}$, respectively), nor in training intensity distribution.

\section{Statistical Analyses}

The Statistical Products of Service Solution package (SPSS Statistics, version 24) was used for all statistical analyses. The preliminary analysis (Kolmogorov-Smirnov) showed that our data did not deviate from a normal distribution for any the considered variables. All descriptive data in text and tables are shown as mean \pm standard deviation (SD). For all participants pooled, a dependent $t$-test was performed to measure the changes from the familiarization test to the pre-test. Thereafter, a $2 \times 2$ repeated measures ANOVA analyses were employed, with type of training (two levels: pre-test and post-test) as a within-subjects factor, and group (two levels: SST and HST) as a between-subjects factor. Independent-samples $t$-tests were used to evaluate between group differences in changes. Pearson correlation analysis was used to investigate the relationship between changes in APO in the 6-s sprint in fresh state and changes in time during the 55-m seated sprint test for each group. We used a Bonferroni correction for all pairwise comparisons in order to prevent Type I error. The corrected/adjusted $p$-value was obtained by dividing the original $\alpha$-value by the number of analyses on the dependent variable. For the rest of the analysis a $p$-value of $\leq 0.05$ was considered statistically significant. The magnitude of changes was also expressed as standardized mean differences (effect size, ES) (Cohen, 1988). ES values between 0.2 and 0.49 indicated a small effect, between 0.5 and 0.79 indicated a moderate effect and 0.8 and above indicated a large effect.

\section{RESULTS}

From the familiarization-test to the pre-test for all participants pooled $(n=28)$, there was a $4.2 \pm 5.6 \%$ increase in PPO $(p=0.001)$ and $3.4 \pm 5.1 \%$ in APO $(p=0.001)$ in the 6-s sprint test in fresh state. Additionally, all participants increased PPO by $3.8 \pm 7.5 \%(p=0.007)$ in the 30 -s sprint test from the 
familiarization-test to the pre-test. No differences was found in other measurements.

\section{Sprint Performance}

In 6-s sprint in fresh state, the SST group increased PPO $(p=0.001)$ and APO $(p=0.001)$ from pre- to post test. No significant changes were observed in the HST group in PPO $(p=0.289)$ and APO $(p=0.158)$ (Table 1). In the 6-s sprints after prolonged cycling, the SST group increased PPO $(p=0.001)$ and APO $(p=0.001)$ from pre- to post test. A significant change was observed in PPO in the HST group $(p=0.030)$ but not in APO ( $p=0.057$ ) (Table 1.) In addition, a significant difference was seen in change between groups from pre- to post-test in PPO and APO both in fresh state $(p=0.023, \mathrm{ES}=0.93$ and $p=0.006, \mathrm{ES}=1.16$, respectively) and after prolonged cycling $(p=0.001, \mathrm{ES}=1.52$ and $p=0.003, \mathrm{ES}=1.42$, respectively) (Table 1).

In the 30-s sprint test, the SST group increased PPO $(p=0.001)$ and APO $(p=0.001)$ from pre- to post test, while no differences were seen in the HST group in PPO $(p=0.065)$ or APO $(p=0.057)$. In addition, a significant differences were seen in change between groups from pre- to post-test in both in PPO $(p=0.015, \mathrm{ES}=1.00)$ and APO $(p=0.032, \mathrm{ES}=0.85)$.

In the $55-\mathrm{m}$ sprint test performed in a seated position, the SST group showed a significant improvement from pre- to post test $(p<0.001)$, while no significant change was observed when the test was performed in a standing positon $(p=0.330)$. No significant changes were observed in the HST group in seated position $(p=0.734)$ or in standing position $(p=0.752)$ In addition, a significant difference was observed between the groups in change from pre- to post test in the seated position test $(p<0.001, \mathrm{ES}=1.97)$ (Table 2$)$. A correlation was also seen in the SST group between improvement in APO in the 6-s sprint test in fresh state and the 55-m seated sprint $(r=-0.74, p=0.036)$.

\section{5-min All-Out Performance}

Both the SST and HST group increased APO from pre- to post test in $5 \mathrm{~min}$ all out performance $(p=0.001$ and $p=0.004$, respectively) (Table 1 ), with no significant difference in change between groups from pre- to post test $(p=0.273$, ES $=0.0 .43$ ).

\section{RM-Squat and Body Composition}

From pre- to post test, the HST group increased load in the 1RM half-squat test by $9.1 \pm 3.7 \%$, from $125 \pm 24 \mathrm{~kg}$ to $136 \pm 25 \mathrm{~kg}$ $(p<0.001)$, while the SST group showed a $3.9 \pm 3.8 \%$ decrease, from $128 \pm 32 \mathrm{~kg}$ to $122 \pm 30 \mathrm{~kg}(p=0.001)$. In addition, a significant difference in change between the groups was observed $(p<0.001, \mathrm{ES}=3.40)$.

No difference within groups was found in body mass $(\mathrm{kg})$ (SST: $p=0.055$, HST: $p=0.339$ ), body fat (\%) (SST: $p=0.856$, HST: $p=0.157)$ or muscle mass (kg) (SMM) (SST; $p=0.302$, HST; $p=0.215)$. Nor was a significant difference in change seen between groups in body mass $(p=0.364$, ES $=0.34)$, body fat $(p=0.398, \mathrm{ES}=0.33)$ or SMM $(p=0.118, \mathrm{ES}=0.61)($ Table 2$)$.

\section{$\dot{\mathrm{V}}_{2 \max }, \mathrm{W}_{\max }$, Power Output at [ $\left.\mathrm{La}^{-}\right]$of $4 \mathrm{mmol} \cdot \mathrm{L}^{-1}$, and Gross Efficiency}

In $\mathrm{VO}_{2 \text { max }}$, the HST group had a significant increase from pre- to post test $(p=0.012)$. No significant changes were observed in the SST group $(p=0.103)$. However, there were no group differences in changes between groups ( $p=0.502, \mathrm{ES}=0.26$ ) (Table 2).

In $\mathrm{W}_{\text {max }}$, the SST group had a significant increase from pre- to post test $(p=0.002)$, whereas no change was observed in the HST group $(p=0.580)$. In addition, a significant difference in change between groups was observed $(p=0.037, \mathrm{ES}=0.91)$.

At $\left[\mathrm{La}^{-}\right]$of $4 \mathrm{mmol} \cdot \mathrm{L}^{-1}$, the HST group significantly increased power output from pre- to post test $(p=0.004)$. No

TABLE 1 | An overview of the results obtained in the 6 and 30-s sprints, and the 5-min all-out test, measured pre and post 6-weeks of short-sprint training (SST) or heavy strength training (HST).

\begin{tabular}{|c|c|c|c|c|c|c|}
\hline & \multicolumn{3}{|c|}{ SST group } & \multicolumn{3}{|c|}{ HST group } \\
\hline & pre & post & $\%$ change & pre & post & $\%$ change \\
\hline \multicolumn{7}{|l|}{ Fresh state } \\
\hline $\mathrm{PPO}\left(\mathrm{W} \cdot \mathrm{kg}^{-1}\right)$ & $17.8 \pm 2.5$ & $18.7 \pm 2.5^{*}$ & $5.6 \pm 3.5$ & $17.1 \pm 2.3$ & $17.3 \pm 2.5$ & $1.9 \pm 5.8^{\#}$ \\
\hline $\operatorname{APO}\left(\mathrm{W} \cdot \mathrm{kg}^{-1}\right)$ & $14.9 \pm 1.8$ & $15.6 \pm 1.8^{*}$ & $4.7 \pm 2.6$ & $14.5 \pm 1.6$ & $14.7 \pm 1.7$ & $1.1 \pm 3.5^{\#}$ \\
\hline $\operatorname{APO}\left(\mathrm{W} \cdot \mathrm{kg}^{-1}\right)$ & $14.4 \pm 1.9$ & $15.3 \pm 2.0^{*}$ & $6.1 \pm 1.8$ & $14.5 \pm 1.6$ & $14.7 \pm 1.8$ & $1.8 \pm 4.2^{\#}$ \\
\hline \multicolumn{7}{|l|}{ 30-s sprint: } \\
\hline $\mathrm{PPO}\left(\mathrm{W} \cdot \mathrm{kg}^{-1}\right)$ & $15.8 \pm 2.2$ & $17.0 \pm 2.30^{*}$ & $8.0 \pm 3.8$ & $15.2 \pm 2.3$ & $15.6 \pm 2.5$ & $2.6 \pm 5.9^{\#}$ \\
\hline $\operatorname{APO}\left(\mathrm{W} \cdot \mathrm{kg}^{-1}\right)$ & $9.6 \pm 0.9$ & $9.9 \pm 0.9^{*}$ & $3.7 \pm 2.8$ & $9.3 \pm 0.7$ & $9.4 \pm 0.7$ & $1.3 \pm 2.5^{\#}$ \\
\hline \multicolumn{7}{|l|}{ 5-min all-out } \\
\hline Standing position (sec) & $7.11 \pm 0.33$ & $7.06 \pm 0.34$ & $-0.7 \pm 2.6$ & $7.12 \pm 0.33$ & $7.10 \pm 0.33$ & $-0.4 \pm 1.5$ \\
\hline
\end{tabular}

Values are mean $\pm S D .{ }^{*}$ Differences within groups $(p<0.05)$; "Difference between groups $(p<0.05)$; PPO, Peak power output; APO, average power output. 
TABLE 2 | Physiological variables measured pre and post 6-weeks of short-sprint training (SST) or heavy strength training (HST).

\begin{tabular}{|c|c|c|c|c|c|c|}
\hline & \multicolumn{3}{|c|}{ SST group } & \multicolumn{3}{|c|}{ HST group } \\
\hline & pre & post & $\%$ change & pre & post & $\%$ change \\
\hline Body mass (kg) & $80.1 \pm 8.7$ & $79.6 \pm 9.1$ & $-0.7 \pm 1.4$ & $78.0 \pm 9.9$ & $78.6 \pm 9.8$ & $-0.3 \pm 1.0$ \\
\hline Body fat (\%) & $12.2 \pm 4.5$ & $12.1 \pm 4.1$ & $-0.1 \pm 1.0 p$ & $12.9 \pm 5.8$ & $12.4 \pm 5.7$ & $0.5 \pm 1.6 p$ \\
\hline SMM (kg) & $40.2 \pm 5.2$ & $40.0 \pm 5.2$ & $-0.6 \pm 2.0$ & $39.0 \pm 4.8$ & $39.3 \pm 5.0$ & $0.7 \pm 2.5$ \\
\hline \multicolumn{7}{|l|}{$\dot{\mathbf{v}}_{2 \max }$ test: } \\
\hline$\dot{\mathrm{VO}}{ }_{2 \max }\left(\mathrm{L} \mathrm{min}^{-1}\right)$ & $5.03 \pm 0.60$ & $5.11 \pm 0.65$ & $1.6 \pm 4.7$ & $4.80 \pm 0.66$ & $4.95 \pm 0.59^{*}$ & $3.4 \pm 5.2$ \\
\hline$\dot{\mathrm{V}} \mathrm{O}_{2 \max }\left(\mathrm{mL} \cdot \mathrm{min}^{-1} \cdot \mathrm{kg}^{-1}\right)$ & $63.3 \pm 5.9$ & $64.8 \pm 6.2$ & $2.5 \pm 4.3$ & $61.1 \pm 6.0$ & $63.4 \pm 6.7^{*}$ & $3.8 \pm 5.5$ \\
\hline $\mathrm{W}_{\max }\left(\mathrm{W} \cdot \mathrm{kg}^{-1}\right)$ & $5.2 \pm 0.5$ & $5.4 \pm 0.5^{*}$ & $3.2 \pm 2.8$ & $5.2 \pm 0.6$ & $5.2 \pm 0.6$ & $0.6 \pm 2.9 \#$ \\
\hline \multicolumn{7}{|l|}{$4 \mathrm{mmol} \cdot \mathrm{L}^{-1}\left[\mathrm{La}^{-}\right]$} \\
\hline Power $\left(\mathrm{W} \cdot \mathrm{kg}^{-1}\right)$ & $3.7 \pm 0.5$ & $3.8 \pm 0.5$ & $2.3 \pm 4.9$ & $3.6 \pm 0.5$ & $3.7 \pm 0.5^{*}$ & $4.3 \pm 5.6$ \\
\hline \multicolumn{7}{|l|}{ Gross Efficiency (\%) } \\
\hline Fresh state (\%) & $20.3 \pm 1.3$ & $20.0 \pm 1.1$ & $-0.3 \pm 0.9 p$ & $20.5 \pm 1.0$ & $20.2 \pm 1.1$ & $-0.3 \pm 0.5 p$ \\
\hline Prolonged cycling (\%) & $19.7 \pm 0.6$ & $19.7 \pm 0.8$ & $0.1 \pm 0.7 p$ & $19.5 \pm 1.0$ & $19.5 \pm 0.7$ & $0.0 \pm 0.7 p$ \\
\hline
\end{tabular}

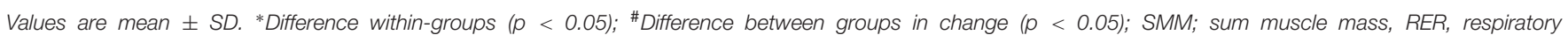

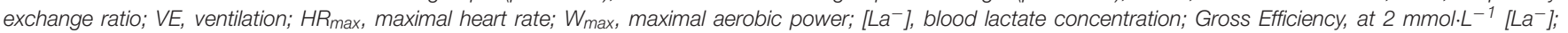
p, percentage point change.

significant change was observed in the SST group $(p=0.060)$. However, no significant difference in change between groups was observed $(p=0.345, \mathrm{ES}=0.37)$.

In GE fresh state and GE after prolonged cycling, there was no significant difference from pre-to post test in neither of the groups (all $p>0.158$ ) (Table 2). In addition, no significant difference in change between groups was observed both in fresh state and after prolonged cycling $(p=0.685$, ES $=0.15$ and $p=0.891, \mathrm{ES}=0.05$, respectively), (Table 2).

\section{DISCUSSION}

The primary aim of the present study was to compare the effects of HST and SST following a 4-week preparation strength-training period on sprint and endurance capacities in trained cyclists. The main findings showed that: (1) 6 weeks of SST induced greater improvement in PPO and APO in 6-s sprint performance both in fresh state and after prolonged cycling, as well as greater improvement in PPO and APO during a 30-s sprint test compared to HST. (2) SST also led to greater improvement than HST in 55-m sprint performance on the cyclists' own bike, with a significant correlation between the improvement in the 6-s sprint performance and gains in the $55-\mathrm{m}$ seated sprint within the SST group. (3) HST induced a larger 1RM improvement than SST in the half-squat test. (4) No group differences were detected in the 5-min all-out test, $\mathrm{VO}_{2 \max }$, GE, or power output at $4 \mathrm{mmol} \cdot \mathrm{L}^{-1}\left[\mathrm{La}^{-}\right]$, while the SST group increased $\mathrm{W}_{\max }$ more than the HST group.

\section{Sprint Performance}

Although both SST and HST are common training methods that intend to improve sprint performance in competing cyclists, this is the first study to compare the effects of these types of training on various factors ranging from sprint to endurance indices and maximal strength. A main finding in the present study was the significantly greater improvement in sprint performance in the SST group compared to the HST group. The positive effects of SST on sprint performance were expected, since previous studies conducted both on untrained (Linossier et al., 1993, 1997) and relatively trained (Jakeman et al., 2012) participants showed similar results. Although improvement after SST is previously shown, this is the first study to show that SST is more effective than continuing HST on the aforementioned sprint performance measures in trained cyclists. The specificity of the SST is the most likely explanation for the greater improvement in the SST group. However, the greater metabolic disturbance caused by SST (Kristoffersen et al., 2018) might be an additional reason for the greater improvement in APO in the 30-s test, where lactic anaerobic capacity is needed (Jacobs et al., 1983; Ross and Leveritt, 2001).

In contrast to our findings, previous studies report increased PPO and APO in well-trained cyclists in 30-s all-out cycling after 10-12 weeks of HST (Ronnestad et al., 2010a, 2016). The lack of improvement in our study might be explained by the shorter intervention period, or by the 4-week preparation strength-training period performed by all participants before the start of the 6-week intervention period. The 4-week strengthtraining period was added in order to reflect common practice in the competitive cyclists' preparatory period. During this 4-week period, increased PPO and APO were seen in the 6-s sprint test in fresh state and in PPO during the 30-s test, indicating that a short duration of strength-training may improve short-sprint performance in trained cyclists. However, sprint performance during the following 6-week intervention did not improve after HST, although this group improved $1 \mathrm{RM}$ in half squat during the same period. Therefore, it is likely that the neuromuscular adaptations relevant to sprinting faster were taken out in the first few weeks with preparatory strength-training, before more specific stimulation through SST was needed to gain further effects on sprinting. The specific effects of HST led solely to higher $1 \mathrm{RM}$ strength, without any indications of hypertrophic response. 
Muscle hypertrophy has been related to improved cycling sprint performance after HST (Ronnestad et al., 2010a; Vikmoen et al., 2016) and the present 6 weeks intervention may have been too short to achieve muscle hypertrophy, at least when combined with a relatively large amount of endurance training (Kraemer et al., 1995). In the future, it should be investigated whether a longer period of SST would further accelerate the effect on sprint performance indices, or if an initial focus on HST, followed by a change in stimulus through more explosive and movementspecific exercise (as experienced by the SST group) is more beneficial. This mix of HST and SST training is indeed interesting, as our findings showed a 3.7\% decrease in strength after 6 weeks of SST, indicating that SST does not satisfactorily maintain any HST-induced strength gain. Therefore, long periods without strength training maintenance should be avoided if cyclists wish to maintain or develop their strength (Ronnestad et al., 2015b).

In this study, we also examined whether SST performed on an ergometer and HST could improve sprint performance on the cyclists' own bike. In previous studies, the association between ergometer cycling and outdoor cycling is not clear, and it has even been suggested that sprint tests should be performed in actual cycling to provide valid assessment of actual changes in performance (Bertucci et al., 2005). In the present study, we showed that 6 weeks of SST on an ergometer increased 55$\mathrm{m}$ sprint performance in actual cycling, while no improvement was seen after HST. Furthermore, we showed strong significant associations between the changes in APO in the 6-s sprint test on the ergometer and in the 55-m seated sprint on the cyclists' own bike in the SST group. This indicate that 6 weeks of SST on an ergometer have great ecological validity for improvement in the field. In contrast, no change was seen when the $55-\mathrm{m}$ sprint was performed standing, confirming the specific significance.

\section{Endurance Performance and Physiological Capacities}

In the 5-min all-out test, the SST and the HST showed significant increases in APO by 5.7 and 3.3\%, respectively, with no significant differences between groups. The 5-min all-out test was chosen as a functional measure of the capacity for very intensive cycling (Hansen et al., 2006; Ronnestad et al., 2011; Vikmoen et al., 2016), with both aerobic and anaerobic contribution. No previous studies have examined the effects of SST on such tests, while improvement has been reported after HST (Ronnestad et al., 2011; Vikmoen et al., 2016). The direct mechanisms behind this study's 5-min APO improvement after SST remain unclear. However, it is well known that adding HST to endurance training can improve endurance performance (Hausswirth et al., 2010; Koninckx et al., 2010; Ronnestad et al., 2010a; Sunde et al., 2010; Aagaard et al., 2011). Increased leg strength may increase peak torque in the pedal stroke, reduce time to peak torque, and reduce the pedaling torque relative to maximal strength (Ronnestad et al., 2015a). This may allow for higher power output and/or increased blood flow, and in turn improve cycling performance (Ronnestad et al., 2011; Barrett-O’Keefe et al., 2012). In addition, strength training may potentially increase the buffer capacity via increased muscle mass, which in turn will lead to improved anaerobic capacity. However, in the present study, neither of the groups increased muscle mass or improved GE. However, a tendency for an increase in $\mathrm{VO}_{2 \text { peak }}$, coinciding with greater training volume throughout the intervention period in both groups, is a more likely explanation for the improvements in 5-min performance found here.

While no differences between the groups were found in 5-min all-out performance, a significant difference between groups was seen in change from pre- to post-test in $\mathrm{W}_{\max }$ with a significantly greater improvement in the SST group. The $\mathrm{W}_{\max }$ tests demand more high-power production, and since the SST group also showed superior improvements in 6 and 30-s performances, neuromuscular characteristics related to high-power production might have contributed. In contrast to our findings, HST has previously proven to also increase $\mathrm{W}_{\max }$ or time to exhaustion at $\mathrm{W}_{\max }$ in cyclists (Hickson et al., 1988; Ronnestad et al., 2010a; Sunde et al., 2010). However, in agreement with our findings, this positive effect was not observed in a previous study with 6 weeks of strength training (Levin et al., 2009). Unfortunately, we did not have a control group, and cannot conclude whether adding HST or SST has a better effect on 5-min all-out cycling than endurance training alone.

Previous studies have reported GE both as unaffected (Aagaard et al., 2011; Ronnestad et al., 2016), reduced (Aagaard et al., 2011) and improved (Sunde et al., 2010; Ronnestad et al., 2011) after HST in cyclists. To the best of our knowledge, no previous studies have investigated how SST affects GE. However, our results showed no change in GE after 6 weeks of SST or HST, or difference in change between groups, neither in a fresh state nor after prolonged cycling.

The present study was conducted during the preparatory period, and as expected, both groups had increased endurance training volume during this period. After 6 week, the HST group had a significant increase in $\dot{\mathrm{V}}_{2 \max }$ and power output at $\left[\mathrm{La}^{-}\right] 4 \mathrm{mmol} \cdot \mathrm{L}^{-1}$, which is in line with previous findings, and indicating that HST don't have any negative interference effects on the endurance capacity (Ronnestad et al., 2010a). However, there were no differences between the groups in $\mathrm{VO}_{2 \max }$, power output at $\left[\mathrm{La}^{-}\right] 4 \mathrm{mmol} \cdot \mathrm{L}^{-1}$ or total training volume after 6 weeks of SST and HST. Therefore, it is likely that the increase in $\dot{\mathrm{V}} \mathrm{O}_{2 \max }$ is caused by changes in endurance training in this period, and not the 6-week intervention of SST or HST.

\section{CONCLUSION}

Short-sprint training caused a larger increase in PPO and APO during 6- and 30-s all-out cycling on the ergometer, as well as in 55-m sprints on cyclists' own bikes compared to the corresponding effects of HST in trained cyclists. In comparison, HST led to a larger increase in maximal strength than SST, while no group differences were found in 5-min allout performance, $\dot{\mathrm{VO}}_{2 \text { max }}$, power output at $4 \mathrm{mmol} \cdot \mathrm{L}^{-1}\left[\mathrm{La}^{-}\right]$, or gross efficiency. Altogether, this shows a high degree of specificity of the implementation of both SST and HST, with SST providing superior speed and power gains whereas HST are most effective for improving strength, and no differences in their influence 
on endurance variables among trained cyclists during a 6-week intervention period. SST performed on an ergometer also seems to transfer to improved sprint performances on the cyclist's own bike, which indicates that SST performed on an ergometer is a valid training method for road cyclists.

\section{DATA AVAILABILITY}

The datasets generated for this study are available on request to the corresponding author.

\section{ETHICS STATEMENT}

Human Subject Research: The studies involving human participants were reviewed and approved by the Norwegian

\section{REFERENCES}

Aagaard, P., Andersen, J. L., Bennekou, M., Larsson, B., Olesen, J. L., Crameri, R., et al. (2011). Effects of resistance training on endurance capacity and muscle fiber composition in young top-level cyclists. Scand. J. Med. Sci. Sports 21, e298-e307. doi: 10.1111/j.1600-0838.2010.01283.x

Barrett-O’Keefe, Z., Helgerud, J., Wagner, P. D., and Richardson, R. S. (2012). Maximal strength training and increased work efficiency: contribution from the trained muscle bed. J. Appl. Physiol. 113, 1846-1851. doi: 10.1152/japplphysiol. 00761.2012

Bertucci, W., Taiar, R., and Grappe, F. (2005). Differences between sprint tests under laboratory and actual cycling conditions. J. Sports Med. Phys. Fitness 45, 277-283.

Cohen, J. (1988). Statistical Power Analysis for the Behavioural Sciences. Hillsdale, NJ: Lawrence Erlbaum Associates.

Faria, E. W., Parker, D. L., and Faria, I. E. (2005). The science of cycling: physiology and training - part 1. Sports Med. 35, 285-312. doi: 10.2165/00007256200535040-00002

Fleck, S. J. (2011). Non-linear periodization for general fitness \& athletes. J. Hum. Kinet. 29A, 41-45. doi: 10.2478/v10078-011-0057-2

Hansen, E. A., Jensen, K., and Pedersen, P. K. (2006). Performance following prolonged sub-maximal cycling at optimal versus freely chosen pedal rate. Eur. J. Appl. Physiol. 98, 227-233. doi: 10.1007/s00421-006-0266-x

Hausswirth, C., Argentin, S., Bieuzen, F., Le Meur, Y., Couturier, A., and Brisswalter, J. (2010). Endurance and strength training effects on physiological and muscular parameters during prolonged cycling. J. Electromyogr. Kinesiol. 20, 330-339. doi: 10.1016/j.jelekin.2009.04.008

Herbert, P., Sculthorpe, N., Baker, J. S., and Grace, F. M. (2015). Validation of a six second cycle test for the determination of peak power output. Res. Sports Med. 23, 115-125. doi: 10.1080/15438627.2015.1005294

Hickson, R. C., Dvorak, B. A., Gorostiaga, E. M., Kurowski, T. T., and Foster, C. (1988). Potential for strength and endurance training to amplify endurance performance. J. Appl. Physiol. 65, 2285-2290. doi: 10.1152/jappl.1988.65.5. 2285

Hopker, J., Myers, S., Jobson, S. A., Bruce, W., and Passfield, L. (2010). Validity and reliability of the Wattbike cycle ergometer. Int. J. Sports Med. 31, 731-736. doi: 10.1055/s-0030-1261968

Jacobs, I., Tesch, P. A., Bar-Or, O., Karlsson, J., and Dotan, R. (1983). Lactate in human skeletal muscle after 10 and $30 \mathrm{~s}$ of supramaximal exercise. J. Appl. Physiol. Respir. Environ. Exerc. Physiol. 55, 365-367. doi: 10.1152/jappl.1983. 55.2.365

Jakeman, J., Adamson, S., and Babraj, J. (2012). Extremely short duration highintensity training substantially improves endurance performance in triathletes. Appl. Physiol. Nutr. Metab. 37, 976-981. doi: 10.1139/h2012-083

Jeukendrup, A. E., Craig, N. P., and Hawley, J. A. (2000). The bioenergetics of World Class Cycling. J. Sci. Med. Sport 3, 414-433. doi: 10.1016/s1440-2440(00) 80008-0
Centre for Research Data (NSD). The patients/participants provided their written informed consent to participate in this study.

\section{AUTHOR CONTRIBUTIONS}

MK: planning, data collection, statistical analysis, and writing. $\varnothing S$ and BR: planning and writing. HG: planning, statistical analysis, and writing.

\section{ACKNOWLEDGMENTS}

The authors express their thanks to the participants for their time and effort.

Koninckx, E., Van Leemputte, M., and Hespel, P. (2010). Effect of isokinetic cycling versus weight training on maximal power output and endurance performance in cycling. Eur. J. Appl. Physiol. 109, 699-708. doi: 10.1007/s00421-010-1407-9

Kraemer, W. J., Patton, J. F., Gordon, S. E., Harman, E. A., Deschenes, M. R., Reynolds, K., et al. (1995). Compatibility of high-intensity strength and endurance training on hormonal and skeletal muscle adaptations. J. Appl. Physiol. 78, 976-989. doi: 10.1152/jappl.1995.78.3.976

Kraemer, W. J., and Ratamess, N. A. (2004). Fundamentals of resistance training: progression and exercise prescription. Med. Sci. Sports Exerc. 36, 674-688. doi: 10.1249/01.mss.0000121945.36635.61

Kristoffersen, M., Sandbakk, O., Tonnessen, E., Svendsen, I., Paulsen, G., Ersvaer, E., et al. (2018). Power production and biochemical markers of metabolic stress and muscle damage following a single bout of short-sprint and heavy strength exercise in well-trained cyclists. Front. Physiol. 9:155. doi: 10.3389/fphys.2018. 00155

Levin, G. T., McGuigan, M. R., and Laursen, P. B. (2009). Effect of concurrent resistance and endurance training on physiologic and performance parameters of well-trained endurance cyclists. J. Strength Cond. Res. 23, 2280-2286. doi: 10.1519/JSC.0b013e3181b990c2

Linossier, M. T., Denis, C., Dormois, D., Geyssant, A., and Lacour, J. R. (1993). Ergometric and metabolic adaptation to a 5-s sprint training programme. Eur. J. Appl. Physiol. Occup. Physiol. 67, 408-414. doi: 10.1007/bf00376456

Linossier, M. T., Dormois, D., Perier, C., Frey, J., Geyssant, A., and Denis, C. (1997). Enzyme adaptations of human skeletal muscle during bicycle shortsprint training and detraining. Acta Physiol. Scand. 161, 439-445. doi: 10.1046/ j.1365-201X.1997.00244.x

Lloyd Jones, M. C., Morris, M. G., and Jakeman, J. R. (2017). Impact of time and work:rest ratio matched sprint interval training programmes on performance: a randomised controlled trial. J. Sci. Med. Sport 20, 1034-1038. doi: 10.1016/j. jsams.2017.03.020

Rhea, M. R., Ball, S. D., Phillips, W. T., and Burkett, L. N. (2002). A comparison of linear and daily undulating periodized programs with equated volume and intensity for strength. J. Strength Cond. Res. 16, 250-255. doi: 10.1519/ 00124278-200205000-00013

Ronnestad, B. R. (2009). Acute effects of various whole body vibration frequencies on 1RM in trained and untrained subjects. J. Strength Cond. Res. 23, 2068-2072. doi: 10.1519/JSC.0b013e3181b8652d

Ronnestad, B. R., Hansen, E. A., and Raastad, T. (2010a). Effect of heavy strength training on thigh muscle cross-sectional area, performance determinants, and performance in well-trained cyclists. Eur. J. Appl. Physiol. 108, 965-975. doi: 10.1007/s00421-009-1307-z

Ronnestad, B. R., Hansen, E. A., and Raastad, T. (2010b). In-season strength maintenance training increases well-trained cyclists' performance. Eur. J. Appl. Physiol. 110, 1269-1282. doi: 10.1007/s00421-010-1622-4

Ronnestad, B. R., Hansen, E. A., and Raastad, T. (2011). Strength training improves 5-min all-out performance following $185 \mathrm{~min}$ of cycling. Scand. J. Med. Sci. Sports 21, 250-259. doi: 10.1111/j.1600-0838.2009.01035.x 
Ronnestad, B. R., Hansen, J., Hollan, I., and Ellefsen, S. (2015a). Strength training improves performance and pedaling characteristics in elite cyclists. Scand. J. Med. Sci. Sports 25, e89-e98. doi: 10.1111/sms.12257

Ronnestad, B. R., Hansen, J., Hollan, I., Spencer, M., and Ellefsen, S. (2015b). In-season strength training cessation impairs performance variables in elite cyclists. Int. J. Sports. Physiol. Perform. 11, 727-735. doi: 10.1123/ijspp.20150372

Ronnestad, B. R., Hansen, J., and Nygaard, H. (2016). 10 weeks of heavy strength training improves performance-related measurements in elite cyclists. J. Sports Sci. 35, 1435-1441. doi: 10.1080/02640414.2016.1215499

Ronnestad, B. R., and Mujika, I. (2014). Optimizing strength training for running and cycling endurance performance: a review. Scand. J. Med. Sci. Sports 24, 603-612. doi: 10.1111/sms.12104

Ross, A., and Leveritt, M. (2001). Long-term metabolic and skeletal muscle adaptations to short-sprint training: implications for sprint training and tapering. Sports Med. 31, 1063-1082. doi: 10.2165/00007256-200131150-00003

Skovereng, K., Sylta, O., Tonnessen, E., Hammarstrom, D., Danielsen, J., Seiler, S., et al. (2018). Effects of initial performance, gross ffficiency and O 2peak characteristics on subsequent adaptations to endurance training in competitive cyclists. Front. Physiol. 9:713. doi: 10.3389/fphys.2018.00713
Sunde, A., Storen, O., Bjerkaas, M., Larsen, M. H., Hoff, J., and Helgerud, J. (2010). Maximal strength training improves cycling economy in competitive cyclists. J. Strength Cond. Res. 24, 2157-2165. doi: 10.1519/JSC.0b013e3181ae b16a

Vikmoen, O., Ellefsen, S., Troen, O., Hollan, I., Hanestadhaugen, M., Raastad, T., et al. (2016). Strength training improves cycling performance, fractional utilization of VO2max and cycling economy in female cyclists. Scand. J. Med. Sci. Sports 26, 384-396. doi: 10.1111/sms. 12468

Conflict of Interest Statement: The authors declare that the research was conducted in the absence of any commercial or financial relationships that could be construed as a potential conflict of interest.

Copyright (c) 2019 Kristoffersen, Sandbakk, Rønnestad and Gundersen. This is an open-access article distributed under the terms of the Creative Commons Attribution License (CC BY). The use, distribution or reproduction in other forums is permitted, provided the original author(s) and the copyright owner(s) are credited and that the original publication in this journal is cited, in accordance with accepted academic practice. No use, distribution or reproduction is permitted which does not comply with these terms. 